\title{
Wearable Integrated Soft Haptics in a Prosthetic Socket
}

\author{
Federica Barontini ${ }^{1,2,3}$, Manuel G. Catalano ${ }^{3}$, Giorgio Grioli ${ }^{3}$, Matteo Bianchi ${ }^{1,2}$ and Antonio Bicchi ${ }^{1,2,3}$
}

\begin{abstract}
Modern active prostheses can be used to recover part of the motor function associated with the loss of a hand. Nevertheless, most sensory abilities are lost, and the person has to manage interaction by relying mostly on visual feedback. Despite intensive research devoted to convey touch related cues, very few solutions have been integrated in a real prosthesis worn by a user. This paper describes a soft pneumatic feedback system designed with integrability and wearability among its main concerns. At the system core, two soft pneumatic actuators are placed in contact with the subject's skin and inflated to provide pressure stimuli, which can be used to represent force exerted by the hand grasping. We report on the design and the characterization of the system, including behavioural experiments with able-bodied participants and one prosthesis user. Results from psychophysical, dexterity and usability tests show that the system has the potential to restore sensory feedback in hand amputees, and can be a useful tool for enabling a correct modulation of the force during grasping and manipulation tasks.
\end{abstract}

\section{INTRODUCTION}

The interaction of humans with the surrounding world is an extremely complex phenomenon, mostly enabled by our hands. These play an active role in the application of forces to external objects, while receiving a large amount of sensing information about the external world through the tactile channels such as objects' softness, dimensions, texture, temperature, and so on [1]. Unfortunately, this incredible biomechanical system can suffer irreversible injuries that prevent an effective implementation of the natural sensorymotor capabilities. In the most severe cases, these can lead to upper extremity amputation, a phenomenon that affects over 2 million people in US only ${ }^{1}$, with a significant increase of the average related healthcare costs (over 150K dollars) with respect to non-amputee population ([2], [3]).

The development of novel myo-electric (electromyography - driven) mechatronic prostheses [4] obtained important results for the restoration of the motoric components, lost with the amputation. However, there are no matching advancements in the sensory domain yet, to restore the role of the hands as information collectors through the haptic channel. This, in turn, translates also in a sub-optimal control performance in everyday use of the prosthesis [5], since the user has to rely only on the exploitation of visual

*This project has received funding from the European Union's Horizon 2020 research and innovation programme under grant agreement No. 810346 (Natural BionicS) and by the Italian Ministry of Education and Research (MIUR) in the framework of the CrossLab project (Department of Excellence).

${ }^{1}$ Centro di Ricerca "Enrico Piaggio", Universitá di Pisa, Largo Lucio Lazzarino 1, 56122 Pisa, Italy federica.barontinil6egmail.com

${ }^{2}$ Dipartimento di Ingegneria dell'Informazione, Universitá di Pisa, Via Caruso 16, 56122 Pisa, Italy

${ }^{3}$ Soft Robotics for Human Cooperation and Rehabilitation, Fondazione Istituto Italiano di Tecnologia, via Morego 30, 16163 Genova, Italy

${ }^{1}$ PhaseSpace, Inc., http://www.biotechpossibilities.com

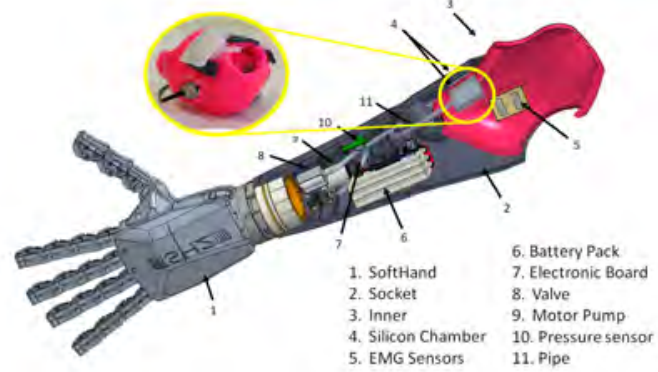

Fig. 1: Overview of the main components of the Wearable Integrated Soft Haptics device.

feedback, which cannot always provide a satisfactory amount of information [6].

Although, the restoration of the haptic channels can be implemented by relying on invasive systems that interface to the user nervous system [7], such solutions are still very experimental. Therefore, much research focuses on alternative non-invasive techniques to restore the haptic channel on the amputates limb. The latter exploit sensory substitution through the usage of wearable haptic devices [8]. These convey touch-mediated information through e.g. skin-stretch, pressure, vibrations or electrical stimulation (see e.g. [9], [10], [11]). An ideal sensory substitution loop should provide [12] modality matching (MM), by delivering artificial cues that share the same sensory modality as the natural sensation, and somatotopic matching (SM), by delivering cues in the same place where they would naturally be felt. Although perfect SM is impossible to achieve on an amputee for obvious reasons, it was observed in [13] that the sensation elicited by contacts at the stump level can be interpreted by the central nervous system as similar to the sensation perceived at the finger level. Ideally a device integrated inside a prosthesis could be able to apply haptic stimuli directly at the stump level, and may effectively overcoming the limitation of previous solutions and satisfy the somatotopic matching paradigm, without invasive solution. Studies focusing on discrete feedback, such as [14], placed pressure sensor thimbles on a prosthetic hand, and used a cuff to convey vibrational stimuli on the upper arm. In [15] the authors used vibrational stimuli to convey physical information of the surface and first-contact cue. Antfolk et al [16] used five controlled pressure devices to transfer tactile input to various locations of the forearm.

In an attempt to go a step further towards the realization of the ideal feedback, we present a novel wearable soft pneumatic force feedback, composed by two silicone chambers, used to exert pressures in specific stump sites. This solution, named Wearable Integrated Soft Haptic device (WISH), strives to achieve both good modality matching (MM) and somatotopic matching (SM). Indeed, according to [12] the 
exertion of a pressure cue has the same modality of stimuli related to those naturally associated to grasping forces, while the application of these stimuli at the stump level provides a good somatotopic approximation, as demonstrated in [13].

Other studies tried to exploit soft robotic technologies to propose a MM and SM stimuli. In [17], the authors present a soft pneumatic actuators able to provide tactile and vibration feedback. In [18], the authors present a passive device based on a glove with silicon bulbs in the fingertips. When they are pressed, the air is transferred via silicone pipes that reached actuators inside the prosthetic socket and gave pressure (mechanotactile feedback) on the skin corresponding with the phantom hand map zones in the residual limb. Despite the system obtained a positive rating, the stimuli provided were not meaningful. Possible reasons could be due to the fact that this is a passive feedback and it is not possible to amplify the signal. Moreover each stimulus was mapped in a single pressure stimulus, and the subject typically missed the perception of the overall pressure. The novelty of the WISH w.r.t. current solutions is the ability to amplify, modulate and convey a pressure stimulus that represents the overall force applied by the robotic hand on an object during the grasp phase. Furthermore it is worth mentioning that WISH can be easily integrated inside the inner socket (see Fig. 1). The paper is organized as follows: the system design is examined in Section II, a mechanical and psychophysical characterization is then provided in Section III, while the prosthesis integration is then described in Section IV. Then, section $\mathrm{V}$ presents the experiments carried out with a prosthetic user to test the WISH device in a real scenario. Finally, section VI critically discusses the results and potential future development of WISH are then proposed in Section VII.

\section{SYSTEM DESIGN}

The Soft Haptic pneumatic system is composed by a mechanical actuation unit powered by a diaphragm pump, and a feedback interface composed by two silicone chambers. We choose a rectangular shape to design the chambers, which have to be integrated into the inner socket. In this manner, we aim at maximizing the surface available in the inner.

\section{A. Material and fabrication method}

The chambers are fabricated with a bio-compatible silicone rubber, in order to guarantee a safe contact with the skin. More specifically, we opted for the silicone Ecoflex $30^{2}$, a platinum-catalyzed silicone, because of its softness (10 psi, 100\% Modulus), and robustness (Tensile Strength 200 psi, and Elongation at Break 900\%). The chambers were fabricated using a custom 3-part mold, shown in Fig 2(a). Finally it is made to incorporate a piece of non-elastic fabric $(0.4 \mathrm{~mm}$ thick cotton 11- count aida cloth - 10 squares per inch) and a second layer of Ecoflex was poured on it. The non-elastic fabric is used as constraint, in order to prevent the inflation of the chamber in both sides. Indeed, our goal is to maximize the inflation of the chamber in contact with the forearm skin. Figure 2(b) show the final result.

\footnotetext{
${ }^{2}$ https: //www. smooth-on.com/products/ecoflex-00-30
}

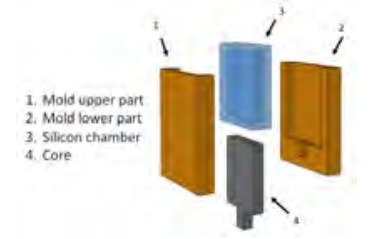

(a)

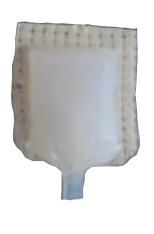

(b)
Fig. 2: Fabrication process of the silicone chambers 2(a). The numbers indicate the components of the mold used to create the chambers. On the right the whole chamber

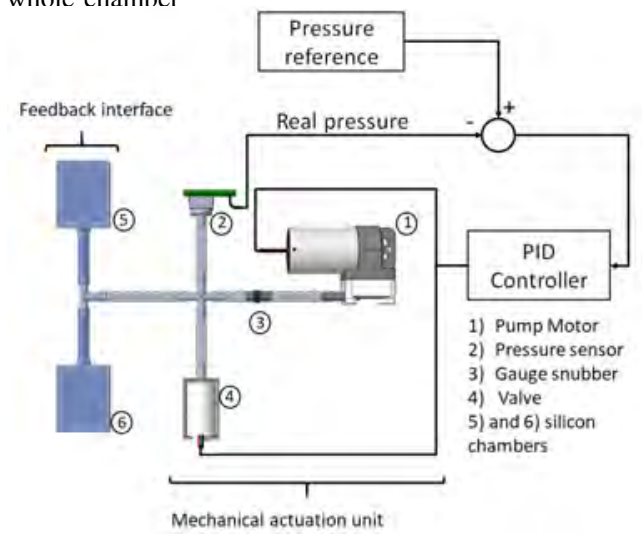

Fig. 3: Overview of the Soft Haptics pneumatic system.

\section{B. The pneumatic system}

Figure 3 shows an overview of the complete haptic system, which is composed by two main subsystem: the feedback interface and the mechanical actuation unit. The feedback interface is composed by two silicone chambers $(5,6$ in Fig 3 ) connected to the mechanical actuation unit with a silicone connecting pipe. The actuation unit is powered by a D220S diaphragm pump (by TSC Micropumps, (1) Fig 3), with a size of $42 \times 27 \times 17 \mathrm{~mm}$, a weight of $31 \mathrm{gr}$, a flow rate of $452(\mathrm{ml} / \mathrm{min})$, and a max continous power of 0.4 Watt. The motor is connected to a solenoid valve (4) KSV05A (rated Voltage of DC $3 \mathrm{~V}$ and a rate current of $75 \mathrm{~mA}$ ), and to an integrated silicone pressure sensor (2) MPXA4250AC6U (Pressure range of $20-250 \mathrm{kPa}$, sensitivity of $20 \mathrm{mV} / \mathrm{kPa}$, an operation temperature from $-40^{\circ}$ to $125^{\circ} \mathrm{C}$, and an accuracy of $\left.\pm 1.5 \% V_{F S S}\right)$. To reduce the pressure oscillation introduced by the pump we use a gauge snubber (3). It is worth noticing that the device presented in this work is only a proof of concept and the primary goal of our work was to explore if the WISH system could be a viable solution to deliver force feedback in prosthetic applications. Future work will consider an in-depth investigation of the technical aspects that are crucial for an everyday life usage of our system, including the characterization of the durability of the silicone chambers as well as of the actuation unit.

\section{Electronic and control system}

We used a custom electronic board based on a Cypress Psoc (Programmable System-on-Chip) micro-controller, with RS485 communication protocol, to control the pump flow rate. The design of the electronic board is open source and more information can be found on the Natural Machine Motion Initiative website ${ }^{3}$. The block diagram in Fig 3

\footnotetext{
${ }^{3}$ https://www.naturalmachinemotioninitiative.com/copy-of-elettronica-v1
} 


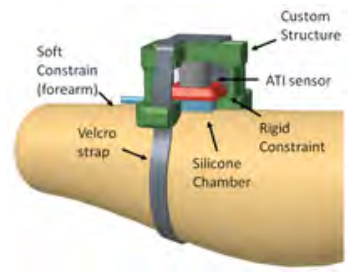

(a)

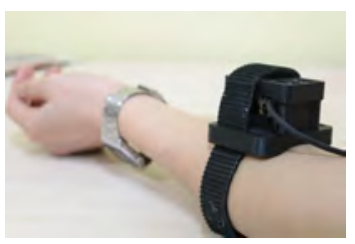

(b)
Fig. 4: The overall architecture and components of the custom structure Fig 4(a). The structure worn by one subject, Fig. 4(b).

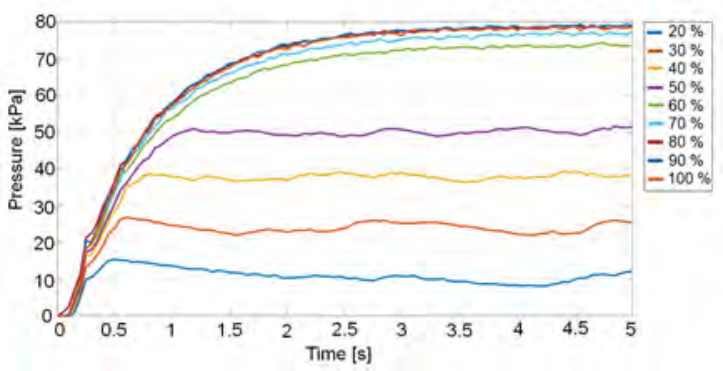

Fig. 5: The picture shows the different levels of pressure obtained with the duty cycle values. The legend, in the picture, indicates all the PWM values considered.

describes the closed loop control scheme. A PID controller modulates the difference between the reference pressure and the pressure readings from the sensor, and activates the pump with a PWM signal. We chose an operation range for the motor between 1 and $3.5 \mathrm{~V}$, which corresponds to a duty cycle value of $20 \%$ and $80 \%$. The choice of the lower limit was mandatory, because the pump does not activate with a duty cycle less than $20 \%$. Whereas we fixed the upper limit to $80 \%$ so to reach a pressure of $25 \mathrm{kPa}$, which is the maximum value we fixed to obtain a normal force on the forearm equal to about 14-15 N (for more details see sec III).

\section{SYSTEM CHARACTERIZATION}

To characterize the system we performed: a physical characterization (sec III-A), and a psychophysical characterization (see III-B) of the pressure just noticeable difference (JND) at different upper limb locations. We also asked participants to undergo a subjective quantitative evaluation to understand the acceptability and effectiveness of the stimulation modality.

\section{A. Physical Characterization}

A 3-axis force sensor, the ATI Gamma $17 \mathrm{Mini}^{4}$, was used to measure the normal force exerted by the WISH device. To be able to collect the data, a custom structure was built to adapt the sensor to the shape of the chamber. We 3D printed, in ABS, two different structures, one where the chamber was completely constrained inside a rigid location, one where the chamber had a rigid constraint on one side and a soft constraint, i.e. the forearm, on the other side.

First we tested the system using different PWM signals, to find a suitable operating range of pressure, which can be perceived on the forearm. We started using a duty cycle of $20 \%$, and increased the value until $100 \%$. We inflated

\footnotetext{
${ }^{4}$ https://www.ati-ia.com/products/ft/ft_models. aspx?id=Mini45
}

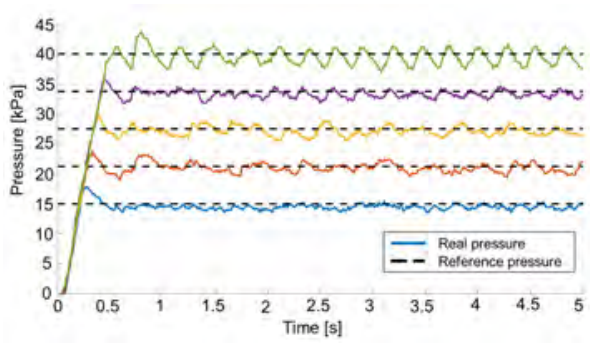

Fig. 6: Pressure evolution within the chamber when a rigid constraint was applied to limit its deformation. The dotted and the colored lines indicate the reference and the real measured pressure.

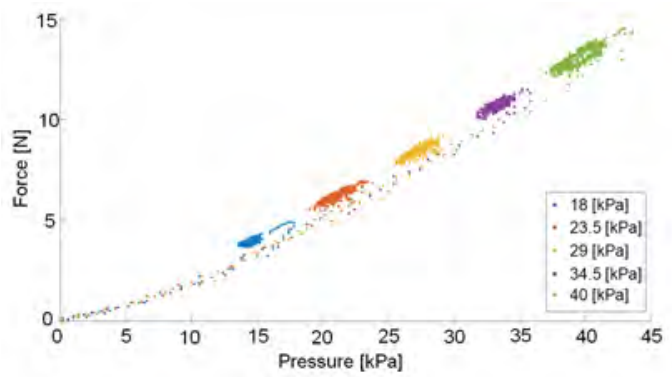

Fig. 7: Force evolution with the different pressure level tested during the characterization.

the chamber for 5 seconds, and then deflated it opening the valve; each duty cycle value was repeated 10 times. Looking at the results obtained from the experiment (see Fig 5), we decided to use a range of pressure between 15 $\mathrm{kPa}$ and $40 \mathrm{kPa}$, because in this range the force had a linear trend respect to different level of pressure (see Fig. 7). We identified five different levels of pressure inside these limits, precisely $15,21.25,27.5,33.75,40 \mathrm{kPa}$, equally spaced, and used the two custom structures for the characterization. The characterization inside the custom rigid structure was accomplished with the silicon chamber completely closed between two rigid constraint on both sides (see Fig 4(a), for the rigid constraint). We inflated the chamber for 5 seconds and then deflated it; each pressure value was repeated for 5 times. We recorded the pressure and the force values corresponding for each inflate/deflate cycle. Figure 6 and 7 show the performance of the PID controller and the force elicited by the chamber on the force sensor.

We repeated the same protocol for the characterization with the soft constraint; in this case one side of the chamber was in contact with the rigid constraint, as before, and the other was directly in contact with the forearm of two subjects. The results obtained show that with the soft constraint, the system is capable to follow precisely the reference pressure. This opens to the possibility to control the pressure to deliver controlled stimuli to the user's forearm.

\section{B. Psychophysical Characterization}

The goal of the Psychophysical characterization was to verify whether the five values of pressure, obtained by the physical characterization, can be clearly distinguished by the subjects. We used the method of the constant stimuli to find the Just Noticeable Difference (JND), as defined in [19], i.e. the minimum amount of pressure that can elicit a different perception in users with respect to a reference stimulus. We considered to place the chambers at two location on the 


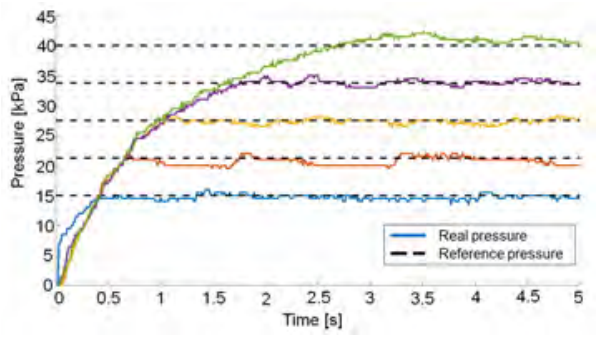

Fig. 8: System performance: the chamber is inside a soft constraint.

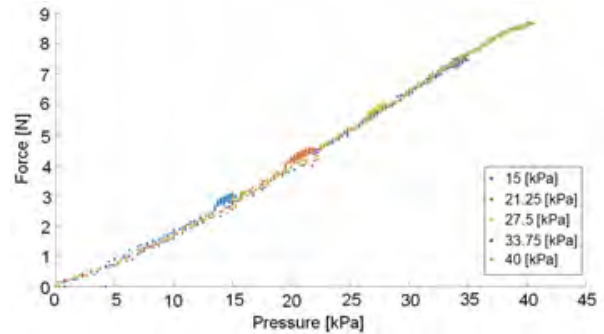

Fig. 9: Force evolution with the chamber inside a soft constraint.

forearm, to ease the integration of the device in different sockets and for different residual limb conditions. In the first configuration, one chamber was placed on the glabrous skin and the other one on the hairy skin, while in the second configuration both the chambers were placed on the hairy skin (see fig 10(a) and 10(b), respectively).

1) Participants: Ten able-bodied participants (one female, mean age 29 and standard deviation of 2.9) took part to the experiment. None of them had experience in the use of soft pressure force feedback devices as sensory substitution techniques. Participants gave their informed consent before the experiment, and nobody had any physical or mental limitation which could have affected the experimental outcome.

2) Stimulus and procedure: The subjects were comfortably seated with their forearm resting on a desk, with the elbow at approximately 90 degree. They wore the WISH device fastened with a Velcro band on their forearm. During the experiment, subjects wore headphones with pink noise to prevent the interference of any auditory cue; in addition they were required not to look at the device during the experiment. The role of the Velcro band is purely instrumental, since it aims at ensuring a proper contact of the silicone chambers with the skin of able-bodied participants. This contact is naturally achieved with prosthesis users thank to integration of the WISH device within the inner socket. Participants received paired stimuli, each stimulus was related to pressure value provided by the chamber on the skin, and were asked to indicate which stimulus in the pair was perceived as higher. Each pair consisted of a reference stimulus (RS) and a comparison stimulus (CS), presented in random order. The pressure was equal to $27.5 \mathrm{kPa}$ in the reference stimulus, and was pseudo-randomly chosen among five discrete and equally spaced values (corresponding to $15,21.25,27.5$, 33.75 , and $40 \mathrm{kPa}$ III-B) in the comparison stimulus. A single trial consisted of: the first stimulus, an inter-stimulus interval $(2 \mathrm{~s})$, and the second stimulus followed by the subject's response. We considered two experimental blocks corresponding to the two locations on the forearm, and the order of execution of the two blocks were counterbalanced

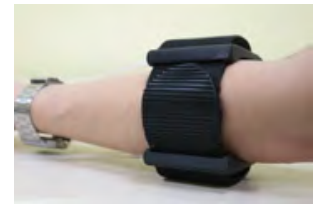

(a)

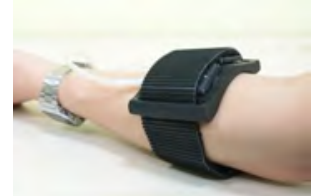

(b)
Fig. 10: The WISH Device applied on a subject's arm (a) first configuration with one chamber on the glabrous skin and one on the hairy skin (bottomup); b) second configuration with both the chambers on the hairy skin only up.

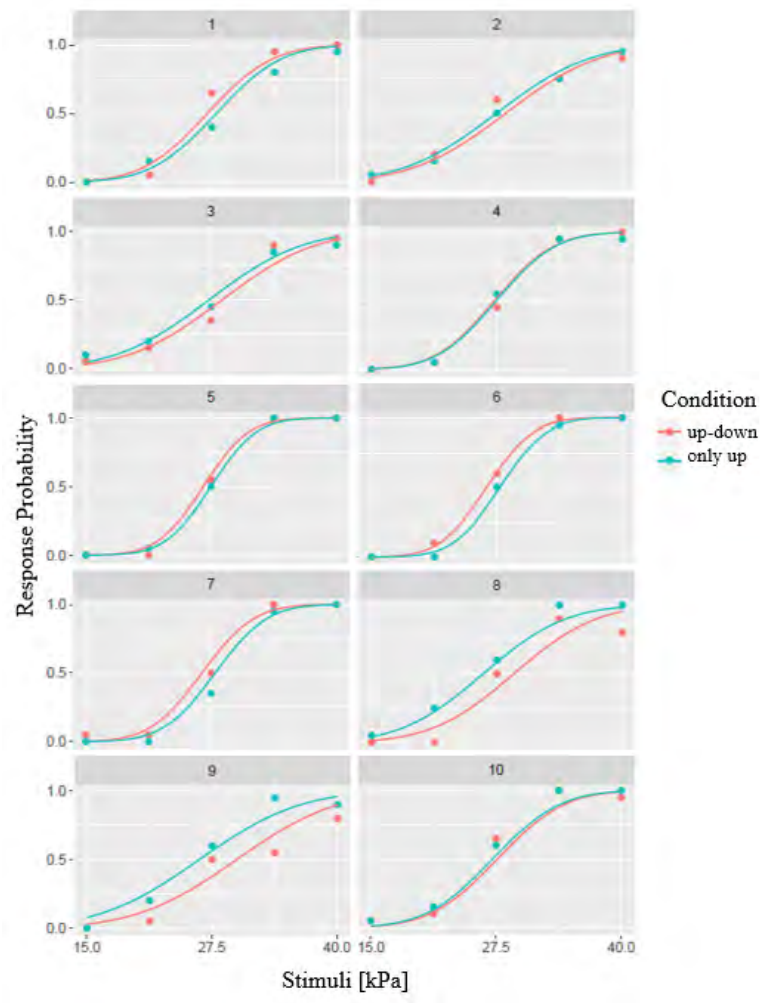

Fig. 11: GLMM fit for all the subjects. Each box represents raw data and model predictions for each single participant (labeled as 1 - 10) in the two condition.

across the subjects. Each block consisted of 100 trials. The binary response of the participants was recorded.

At the end of the experiment, participants underwent through a subjective quantitative evaluation procedure based on a seven point Likert-scale survey, which consisted of questions about the system and the experimental tasks. Participants had to answer by assigning a score ranging from 1 totally disagree to 7 totally agree. This represents a common procedure to evaluate devices for assistive robotics and Human-Robot Interaction [20]. The questionnaire considered the comfort and usability of the experimental setup (Q1, Q2, Q3, Q4 and Q7), the perceived performance (Q5, Q6, Q9 and Q10), and the engagement of the subject (Q8).

3) Data analysis: For the two experimental condition, i.e. the body location for stimulus delivery, we positioned the chambers: 1) one in the glabrous skin and the other on the hairy skin - hereinafter referred to as condition $u p$ down -, and 2) both on the hairy skin - hereinafter referred to as condition only up). We used a Generalized Mixed Model GLMM to determine JND through all the participants [21], [9]. This model enabled cluster data analysis, in our 
Q1 It was easy to wear and use the WISH device

Q2 I was feeling uncomfortable usign the WISH

Q3 I was well-isolated from the external noises during the experiments

Q4 I was able to hear the sounds made by the actuator of the device

Q5 The stimuli provided by the cutaneous device allowed to discriminate different pressure levels $\quad 6.4 \quad 0.69$

Q6 I felt hampered by the air pressure stimuli

Q7 I was able to see the device during the experiment

Q8 At the end of the experiment I felt tired

Q9 I prefer to receive the stimuli at two different body locations, i.e. glabrous and hairy skin

TABLE I: These statements, presented in random order, were rated by the able-bodied subjects using a 7-point Likert scale (1: Strongly disagree, 7: Strongly agree). Means and standard deviations across all individuals are reported.

case a cluster is the collection of repeated responses in several participants. GLMM is a hierarchical model that provides both predictions on the experimental effects, which are assumed to be systematic across participants, and an estimate of the variability between participants. The expected value of the response can be expressed as

$$
\Phi^{-1}\left[P\left(Y_{j}=1\right)\right] \sim \beta_{0}+\beta_{1} x_{j}
$$

where $\left[P\left(Y_{j}=1\right)\right]$ is the probability that, in trial $j$, the participant reported a larger stimulus in the comparison than in the reference stimulus, $\Phi^{-1}[\cdot]$ is the probit transformation of the response probability (i.e., the inverse function of the cumulative normal distribution), and $x_{j}$ is the explanatory variable, i.e. the pressure value provided to the chambers. $\beta_{0}$ and $\beta_{1}$ are the fixed effect parameters, i.e. the intercept and the slope of the linear function (linear predictor), which are the same for all the subjects. For each experimental condition, we estimated the Just Noticeable Difference (JND), i.e. the amount of stimulus change to detect the just noticeable difference, the Point of Subjective Equality (PSE), i.e. the stimulus value yielding a response probability of 0.5 , with the related $95 \%$ confidence intervals (CIs), using the bootstrap method [21].

4) Results: The GLMM fitting to the data is illustrated in Figure 11. The JND was equal to $3.40 \mathrm{kPa}(95 \%$ CIs: $2.72-4.15 \mathrm{kPa})$ for the up-down condition, and $3.38 \mathrm{kPa}$ (95\% CIs: $2.75-4.20 \mathrm{kPa}$ ) for the only up condition. The PSE was equal to $27.51 \mathrm{kPa}(95 \%$ CIs: $26.65-28.46 \mathrm{kPa})$ for the up-down condition and $27.47 \mathrm{kPa}$ (95\% CIs: $26.66-$ $28.47 \mathrm{kPa}$ ) for the only up condition. Results of the bootstrap method revealed that the $95 \% \mathrm{CI}$ of the differences between JNDs and PSEs in the two conditions include zero. These results imply that these differences are not significant.

\section{INTEGRATION IN A PROSTHESIS}

According to the results obtained from the experiment with able-bodied subjects, we integrated the system inside a inner socket positioning both the silicone chambers on the hairy skin (i.e. the only-up configuration of able-bodied subjects); Figure 1 shows the WISH device inside the inner socket. The mechanical structure is composed of 3 main parts: the structural frame, the mechanical actuation and the feedback interface. The structural frame is composed of the SoftHand Pro [22], the socket and the inner socket inclusive of surface electromyographyc (sEMG) sensors (respectively parts $\{1,2,3,5\}$ in Fig. 1). The mechanical part is composed

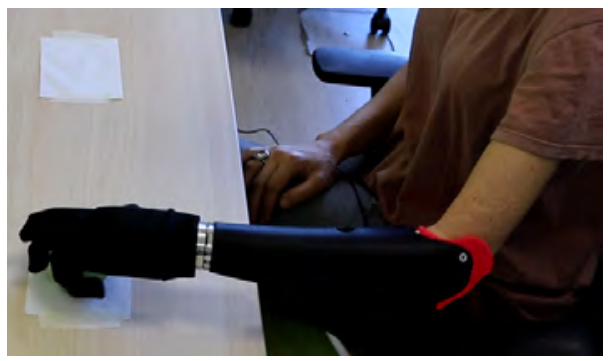

Fig. 12: A prosthetic user wearing the WISH device integrated inside the prosthesis.

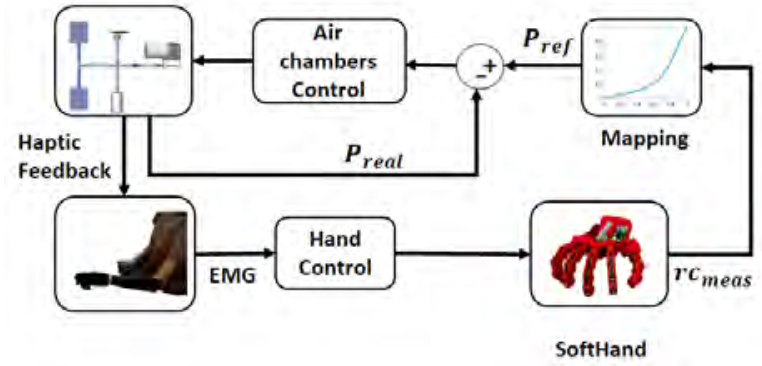

Fig. 13: Block diagram of the SoftHand Pro, controlled by the sEMG sensors, with the haptic feedback in the loop.

of a battery pack (6), an electronic board (7), a solenoid valve (8), a motor pump (9), and a pressure sensor (10). The feedback interface is composed of the two silicone chambers (4) integrated in the upper part of the inner socket (see fig 14). All the components are connected with silicone pipes (10).

\section{A. The control strategy}

The block diagram in Fig. 13 describes the closed-loop control scheme of the SoftHand Pro with the human in the loop. The electromyographic activity from the Flexor Digitorum Superficialis (FDS) and the Extensor Digitorum Communis (EDC) muscles is measured from the skin of the users forearm using two Ottobock 13E200 sensors ${ }^{5}$, which directly provide an amplified, bandpass-filtered, and rectified version of the raw sEMG signals. The reference pressure, $p_{\text {ref }}$, used to control the air pump and the valve is set to be proportional to an estimate of the grasp force exerted by the hand. To estimate the grasp force we used the difference between the estimated current and the real current absorbed by the SoftHand Pro motor, called residual

${ }^{5}$ Otto Bock HealthCare GmbH, http://www.ottobock.com/ 

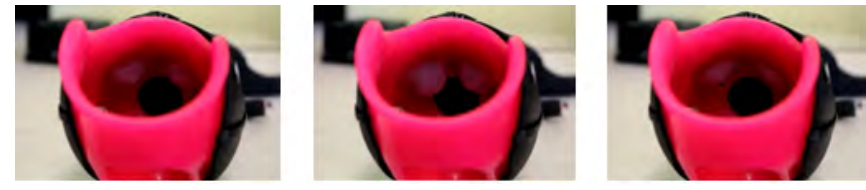

Fig. 14: Sequence of images that show the mode of operation of the chamber inside the inner socket.

\begin{tabular}{lcc}
\hline Comparison & I $>$ ref & $\%$ Success \\
\hline $1-3$ & 0 & $100 \%$ \\
$2-3$ & 5 & $75 \%$ \\
$3-3$ & 11 & $55 \%$ \\
$4-3$ & 11 & $55 \%$ \\
$5-3$ & 16 & $90 \%$ \\
\hline
\end{tabular}

TABLE II: The table reports the results of the comparison between the pair stimuli with the percentage of success.

current, $r c_{\text {meas }}$ (see [23] for details). The tactile stimulation performed by the WISH, directly depends on the value of the SoftHand Pro residual current; this information can be used to understand how much force the hand is applying during the grasp of an object. We establish two thresholds: if the residual current is over $500 \mathrm{~mA}$, it is saturated to this value, the air pump is on, the valve is closed and the chambers start to inflate; if the residual current is equal or minus than zero, the value is set to zero, the pump is off, the valve is open and the chambers are deflated. Mapping the information coming from the robotic hand into feedback device, means to associate each value of the hand with a corresponding pressure value of the device. It is not an on/off control but a real time modulation of the residual current value of the SoftHand into inflation/deflation of the silicone chambers. In our investigation we tried different types of mapping [9], and the best mapping we found out was a parabolic mapping. We imposed three simple constraints: $r c_{\text {meas }}=0$ corresponds to $p_{\text {ref }}=0, r c_{\text {meas }}=r c_{\text {max }}$ corresponds to $p_{\text {ref }}=p_{\text {max }}$, being $r c_{\max }$ the maximum value of the residual current of the SoftHand, and $p_{\max }$ the maximum pressure value inside the chambers; and $r c_{\text {meas }}=\frac{1}{2} r c_{\max }$ was mapped to $p_{\text {ref }}=\frac{4}{5} p_{\max }$. The reference position was:

$$
p_{\text {ref }}=\frac{-30 x^{2}+55 * 450 x}{450^{2}}
$$

where $x=r c_{\text {meas }}-50.0$.

\section{PILOT EXPERIMENTS}

We tested the integrated system with one amputee subject (female, age 39 with agenesis of left forearm). The participant did not suffer from any cognitive impairment, that could have interfered with her ability to follow the instruction of the study. The subject wore her prosthesis, with the WISH integrated, on her left arm, and headphones with pink noise to prevent any auditory cues. Before starting doing other experiments, we accomplish the JND test also with the amputee subject, to verify if the perception of an amputees subject is comparable to the perception of an able-bodied subject. The protocol adopted was equal to the protocol used for the able-bodied participants. In Table II we show the percentage of accuracy of the experiment. We did not perform any statistical analysis, since we have only one subject. At the end of the experiment the subject underwent

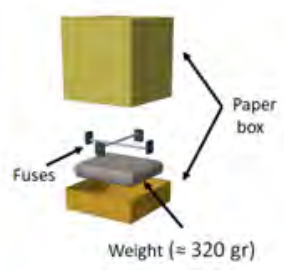

(a)

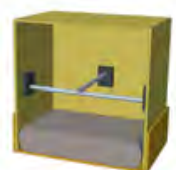

(b)

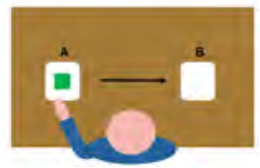

(c)
Fig. 15: Overview of the virtual egg 15(a) and 15(c) and the setup used for the second task

through the subjective quantitative evaluation procedure, the Likert-scale survey (see table III). We asked the subject to accomplish two different task: 1) a cylinder discrimination task, and 2) a virtual eggs task. In our hypothesis what the user really feels is the change in impedance of the chamberskin-muscle system interpose between the rigid socket and the rigid bone, which can be probed by muscle activation. In addition, the diameter of the residual limb of the prosthesis user did not change noticeably when the muscle was relaxed or contracted (remaining approximately equal to $19.5 \mathrm{~cm}$ ). For these reasons, we decided to not explicitly model force changes due to muscle size variation for this subject. This represent an important aspect to be considered in future investigations. These two experiments were designed to evaluate the effectiveness of the feedback provided by the WISH device when using the SoftHand Pro. At the end of both experiments the subject underwent the System Usability Scale (SUS) the most widely used standardized questionnaire for the assessment of perceived usability [24].

\section{A. Cylinder discrimination task}

For the cylinder-softness discrimination task, subject was comfortably seated in front of the table with the hand placed on the desk in a fixed position. The experiment lasted roughly one hour and consisted of two phases. First the training phase, which lasted approximately 20 minutes, the subject made manipulation tasks of different objects of various size, i.e. building pyramids with toy blocks, grasping and moving cylinders. Neither vision nor hearing were obstructed during this phase. During the test phase, which lasted more or less 30 minutes, the subject was blindfolded and wore headphones with pink noise, to prevent any auditory cue. For the test, we selected three stimuli: the hand closed without object, and with a rigid and soft cylinder both, $60 \mathrm{~mm}$ in diameter. For convenience, the three cases were labelled as cylinder 0,1 and 2, respectively. In each trial, we provided participant with two stimuli, sequentially, and we asked her to report which one was the higher pressure. Each cylinder was positioned on the palm of the SoftHand Pro and the subject had to voluntarily close the hand to grasp it. Each pair was presented twice. The test was performed in two modalities, with and without feedback.

\section{B. Virtual eggs task}

We asked to the subject to accomplish a task similar to a Box and Block test: she had to move a virtual egg, from point A to point B, for ten times, without breaking it. This task was used in [25] to assess the grasping efficiency. The virtual egg 
Q1 It was easy to wear and use the WISH device together with the SoftHand.

Q2 I was feeling uncomfortable while using the WISH device together with the SoftHand.

Q3 I was well-isolated from the external noises during the experiments

1

Q4 I was able to hear the sounds made by the actuator of the device

Q5 The stimuli provided by the cutaneous device allowed to discriminate different pressure levels

Q6 At the end of the experiment I felt tired

Q7 I felt hampered by the air pressure stimuli

Q8 I was able to see the device during the experiment

Q9 The sensation provided by the air pump on the forearm felt pleasant

Q10 The sensation provided by the air pump on the forearm felt unpleasant.

Q11 I had the feeling of performing better while receiving force feedback by the air pressure chambers

Q12 I had the feeling of performing better when I was not receiving any force feedback by the air pressure chambers

7

1

3

1

2

6

2

6

TABLE III: These statements, presented in random order, were rated by the amputee subject using a 7-point Likert scale (1: Strongly disagree, 7: Strongly agree). Only the scores are reported.

\begin{tabular}{lcccc}
\hline \multirow{2}{*}{ Trial } & \multicolumn{2}{c}{ Without WISH } & \multicolumn{2}{c}{ With WISH } \\
\cline { 2 - 5 } & Time to move [s] & Broken eggs & Time to move [s] & Broken eggs \\
\hline Task 1 & 6.42 & 1 & 5.70 & 0 \\
Task 2 & 6.56 & 1 & 5.99 & 1 \\
Task 3 & 6.58 & 0 & 8.08 & 0 \\
Task 4 & 7.4 & 1 & 8.02 & 0 \\
Task 5 & 5.92 & 1 & 5.98 & 1 \\
Task 6 & 6.34 & 0 & 7.41 & 1 \\
Task 7 & 4.10 & 1 & 5.29 & 0 \\
Task 8 & 9.97 & 0 & 5.60 & 0 \\
Task 11 & 6.24 & 0 & 5.81 & 0 \\
Task 10 & 8.12 & 1 & 5.77 & 0 \\
\hline
\end{tabular}

TABLE IV: The table reports the time (in second) to move the fragile objects from point $\mathrm{A}$ to point $\mathrm{B}$.

is a box made by paper, dimension $50 \times 50 \mathrm{~mm}$ containing a fragile fuse placed in the middle of the box, and a weight of 320 gr. The fragile fuse is a piece of spaghetto, fragile enough to be broken by an excessively tight pinch grasp. More information regarding the virtual eggs can be found on the Natural Machine Motion Initiative website ${ }^{6}$. The subject stood in front of the table and repeated the experiment with and without the feedback; in both condition there were not limit of time to move the box. For each modality we recorded: 1) the time spend to move the fragile object from A to B (we started recording time when the subject touched the virtual egg and stopped when she released the box), 2) the number of regrip, and 3) the number of broken eggs. Table IV shows the result of the experiment.

\section{Results}

The results of the cylinder-softness discrimination task show a major accuracy when the subject accomplishes the experiment with the device, respect to the condition without feedback. For the cylinder discrimination task, we obtained an accuracy of $100 \%$ for the modality with the feedback, and an accuracy of $75 \%$ for the modality without the feedback.

Table IV shows the results of the fragile object. Although we cannot make any statistical claim, the number of broken eggs without feedback is 6, compared to the 3 with the feedback. Finally from the SUS questionnaire, emerged a positive rate with a total score of 70 , which means a good device but it needs to be improved.

\footnotetext{
${ }^{6} \mathrm{https}: / / \mathrm{www}$.naturalmachinemotioninitiative.com/fragile-objectbenchmark
}

\section{DISCUSSION}

The experimental outcome with the able-bodied and the limb loss participants show that the WISH device can be successfully used to convey the force feedback. From the psychometric experiment and the Likert scale of able-bodied participants, emerges that there is no difference detecting the pressure in different parts of the forearm (questions Q9 and Q10 with mean values of 5.6 and 4.5, and a standard deviation 0.96 and 1.34). In general, subjects felt comfortable to wear and use the device (question Q1 and Q2 with a mean value of 6.5 and 1.3 and a standard deviation of 0.42 and 0.69). During all the experiments, subjects were well isolated from external noises (Q3, Q4 and Q7). The stimuli provided by the device were clear and allowed to discriminate different pressure levels (Q5). Regarding the intuitiveness of the sensory substitution, no one of the participants felt hampered by the air pressure stimuli (Q6 with a mean of 1.3 and a standard deviation of 0.69). At the end of the experiment the participants were not tired (question Q8, mean value 1.6 and stardard deviation of 0.69 ). The results obtained during the experiments with the limb loss participant are very promising. The survey outcome shows that the WISH is easy to wear $(\mathrm{Q} 1)$ meaning that the device is not an obstacle during the wear phase of the prosthesis. Moreover, the subject was not hampered by the air pressure stimuli, and the sensations provided were pleasant (Q7, Q9 and Q10), so we can assume that the MM paradigms is successfully satisfied. The sensation provided to the residual limb is helpful and clear to understand (Q12). The subject was able to recognize different level of pressure during passive and active task (i.e. the cylinder-softness and virtual egg task respectively). During the active task, the subject, using the device, was able to modulate the force in order to grasp the box without broke the fuse inside it. The score of SUS questionnaire underline a positive outcome.

\section{CONCLUSIONS}

We presented the WISH device, a pneumatic force feedback used to convey information on the grasp force of a prosthetic hand that can be integrated in the socket. The psychometrics analysis shows that there is no difference in chamber placement for the two forearm locations that were tested. Experimental outcomes with the prosthetic user reveal a good level of accuracy for the softness discrimination task, 
and a good control of the modulation of the grasping force during manipulation tasks. In conlusion, the WISH device seems to be a viable solution to provide a force feedback completely integrated inside a socket. Future work will focus on further testing with real prosthesis users, including a deeper investigation of the effect of muscle size variations with contraction. While a 1D force feedback output is sufficient for the purpose of the current work (to deliver force-related information based on an indirect estimation of the overall grasping force of the SoftHand), in the future we will consider other prosthetic hands (including versions of the SoftHand with additional degrees of actuations [26]), where multi-dimensional force feedback could be useful. A possible direction include the use of more chambers, as proposed in [18], controlled by multiple air pumps. Another way could be to combine the air pressure with particle jamming, as explored by Stanley et al (2015), to model the output feedback according to different directions.s

\section{ACKNOWLEDGMENT}

The authors would like to thank Nicoletta Colella, Cristiano Petrocelli and Mattia Poggiani for their useful help in the characterization process and system design.

\section{REFERENCES}

[1] D. Katz, The world of touch. Psychology press, 2013.

[2] E. Biddiss, D. Beaton, and T. Chau, "Consumer design priorities for upper limb prosthetics," Disability and rehabilitation: Assistive technology, vol. 2, no. 6, pp. 346-357, 2007.

[3] N. Gurari, K. J. Kuchenbecker, and A. M. Okamura, "Perception of springs with visual and proprioceptive motion cues: Implications for prosthetics," IEEE Transactions on Human-Machine Systems, vol. 43, no. 1, pp. 102-114, 2012.

[4] C. Piazza, G. Grioli, M. Catalano, and A. Bicchi, "A century of robotic hands," Annual Review of Control, Robotics, and Autonomous Systems, vol. 2, no. 1, pp. 1-32, 2019. [Online]. Available: https://doi.org/10.1146/annurev-control-060117-105003

[5] H. Culbertson, S. B. Schorr, and A. M. Okamura, "Haptics: The present and future of artificial touch sensation," Annual Rev. of Control, Robotics, and Autonomous Systems, vol. 1, pp. 385-409, 2018.

[6] C. Fraser and A. Wing, "A case study of reaching by a user of a manually-operated artificial hand," Prosthetics and orthotics international, vol. 5, no. 3, pp. 151-156, 1981.

[7] C. M. Oddo, S. Raspopovic, F. Artoni, A. Mazzoni, G. Spigler, F. Petrini, F. Giambattistelli, F. Vecchio, F. Miraglia, L. Zollo et al., "Intraneural stimulation elicits discrimination of textural features by artificial fingertip in intact and amputee humans," Elife, vol. 5, p. e09148, 2016.

[8] P. Svensson, U. Wijk, A. Bjrkman, and C. Antfolk, "A review of invasive and non-invasive sensory feedback in upper limb prostheses," Expert Review of Medical Devices, vol. 14, no. 6, pp. 439-447, 2017, pMID: 28532184. [Online]. Available: https: //doi.org/10.1080/17434440.2017.1332989

[9] M. Rossi, M. Bianchi, E. Battaglia, M. G. Catalano, and A. Bicchi, "Happro: a wearable haptic device for proprioceptive feedback," IEEE Trans on Biomedical Engineering, vol. 66, no. 1, pp. 138-149, 2018.

[10] A. A. Stanley and K. J. Kuchenbecker, "Evaluation of tactile feedback methods for wrist rotation guidance," IEEE Transactions on Haptics, vol. 5, no. 3, pp. 240-251, 2012.

[11] J. P. Clark, S. Y. Kim, and M. K. OMalley, "The rice haptic rocker: Comparing longitudinal and lateral upper-limb skin stretch perception," in Int. Conference on Human Haptic Sensing and Touch Enabled Computer Applications. Springer, 2018, pp. 125-134.

[12] K. Kim and J. E. Colgate, "Haptic feedback enhances grip force control of semg-controlled prosthetic hands in targeted reinnervation amputees," IEEE Transactions on Neural Systems and Rehabilitation Engineering, vol. 20, no. 6, pp. 798-805, 2012.

[13] C. Antfolk, A. Björkman, S.-O. Frank, F. Sebelius, G. Lundborg, and B. Rosen, "Sensory feedback from a prosthetic hand based on airmediated pressure from the hand to the forearm skin," Journal of rehabilitation medicine, vol. 44, no. 8, pp. 702-707, 2012.
[14] F. Clemente, M. D’Alonzo, M. Controzzi, B. B. Edin, and C. Cipriani, "Non-invasive, temporally discrete feedback of object contact and release improves grasp control of closed-loop myoelectric transradial prostheses," IEEE Transactions on Neural Systems and Rehabilitation Engineering, vol. 24, no. 12, pp. 1314-1322, 2015.

[15] S. Fani, K. Di Blasio, M. Bianchi, M. G. Catalano, G. Grioli, and A. Bicchi, "Relaying the high-frequency contents of tactile feedback to robotic prosthesis users: Design, filtering, implementation, and validation," IEEE Robotics and Automation Letters, vol. 4, no. 2, pp. 926-933, 2019.

[16] C. Antfolk, M. Dalonzo, B. Rosén, G. Lundborg, F. Sebelius, and C. Cipriani, "Sensory feedback in upper limb prosthetics," Expert review of medical devices, vol. 10, no. 1, pp. 45-54, 2013.

[17] J. J. Huaroto, E. Suarez, H. I. Krebs, P. D. Marasco, and E. A. Vela, "A soft pneumatic actuator as a haptic wearable device for upper limb amputees: Toward a soft robotic liner," IEEE Robotics and Automation Letters, vol. 4, no. 1, pp. 17-24, 2018.

[18] U. Wijk, I. K. Carlsson, C. Antfolk, A. Bjrkman, and B. Rosn, "Sensory feedback in hand prostheses: A prospective study of everyday use," Frontiers in Neuro., vol. 14, p. 663, 2020. [Online]. Available: https://www.frontiersin.org/article/10.3389/fnins. 2020.00663

[19] G. A. Gescheider, Psychophysics: the fundamentals. Psychology Press, 2013.

[20] D. Prattichizzo, F. Chinello, C. Pacchierotti, and M. Malvezzi, "Towards wearability in fingertip haptics: a 3-dof wearable device for cutaneous force feedback," IEEE Transactions on Haptics, vol. 6, no. 4, pp. 506-516, 2013.

[21] A. Moscatelli, M. Mezzetti, and F. Lacquaniti, "Modeling psychophysical data at the population-level: the generalized linear mixed model," Journal of vision, vol. 12, no. 11, pp. 26-26, 2012.

[22] S. B. Godfrey, K. D. Zhao, A. Theuer, M. G. Catalano, M. Bianchi, R. Breighner, D. Bhaskaran, R. Lennon, G. Grioli, M. Santello et al., "The softhand pro: Functional evaluation of a novel, flexible, and robust myoelectric prosthesis," PloS one, vol. 13, no. 10, 2018.

[23] D. Mura, M. Barbarossa, G. Dinuzzi, G. Grioli, A. Caiti, and M. G. Catalano, "A soft modular end effector for underwater manipulation: A gentle, adaptable grasp for the ocean depths," IEEE Robotics \& Automation Magazine, vol. 25, no. 4, pp. 45-56, 2018.

[24] J. R. Lewis, "The system usability scale: past, present, and future," International Journal of Human-Computer Interaction, vol. 34, no. 7 , pp. 577-590, 2018.

[25] L. F. Engels, L. Cappello, and C. Cipriani, "Digital extensions with bi-axial fingertip sensors for supplementary tactile feedback studies," in 2018 7th IEEE International Conference on Biomedical Robotics and Biomechatronics (Biorob), 2018, pp. 1199-1204.

[26] C. Della Santina, C. Piazza, G. Grioli, M. G. Catalano, and A. Bicchi, "Toward dexterous manipulation with augmented adaptive synergies: The pisa/iit softhand 2," IEEE Transactions on Robotics, vol. 34, no. 5, pp. 1141-1156, 2018. 